\title{
Stochastic generation of multiscale 3D pore network models of building materials
}

\author{
Steven Claes $^{1, *}$, and Hans Janssen ${ }^{1}$ \\ ${ }^{1}$ KU Leuven, Department of civil engineering, Building physics section, Kasteelpark arenberg 40, 3000 Leuven, Belgium
}

\begin{abstract}
The current experimental determination of hygric properties of porous building materials are demanding in time and effort, merge ad- and desorption techniques, fuses static and dynamic methods, and finally do not yield complete nor robust results. Therefore, numerical pore-scale-based prediction of the hygric properties of building materials is on the rise as an alternative. For building materials, this is mostly based on pore network modelling (PNM), given that these are more efficient compared to lattice Boltzmann or particle hydrodynamic methods. Pore network modelling however requires data of the complete pore network for the building material. With the currently available characterization and visualization techniques, this cannot be readily obtained, as the pore sizes in building materials often span several spatial scales. The aim of this paper is to present a scale invariant stochastic generation. To realize this objective, distributions of direct parameters (pores' sizes, shapes, positions, connections, ...) as well as indirect parameters (overall pore size distribution and open porosity value) are derived from the input data obtained by micro-CT and FIB-SEM and subsequently applied to generate a complete pore network of the porous building material. The quality of the generated PNMs is assessed by comparing them to the original PNMs.
\end{abstract}

\section{Introduction}

Flow and transport phenomena in porous media play a significant role in various fields of science and technology, comprising a spectrum from medical sciences over material sciences to soil and rock sciences. In order to determine the moisture behavior of building components, numerical simulation models are commonly used. However, these models require a good description of the moisture retention and moisture permeability functions, as these are crucial input parameters. The experimental characterization of these hygric properties is impaired by serious weaknesses [1]. The procedure merges ad- and desorption as well as static and dynamic methods, thus ignoring hysteresis, air entrapment and dynamic effects. The measured outcomes additionally remain incomplete, as they do not provide much information in the mid-saturation ranges. Moreover, it necessitates weeks of experiments, and of course, requires to have the actual material at hand. This multifactorial group of flaws implies that hygric analysis of existing materials is cumbersome and time intensive.

In order to overcome these deficiencies, a new approach is currently being introduced in building physics. Pore network models (PNMs) have already proven to be useful to determine fluid flow properties in other research fields, such as geological applications [2], fuel cells [3] and drying technology [4], to determine fluid flow properties. PNMs try to represent the studied pore structure as accurately as possible while retaining a certain simplicity by representing the pore space as a network of pore bodies and pore throats. Hence, the PNM tries to capture local features of the pore-space which are important for the fluid storage and transport processes under investigation. Subsequently these PNMs are subjected to invasion algorithms that mimic different (de)saturation routes: absorption, desorption, imbibition and drying. Once the moisture distribution at a certain capillary pressure has been determined, a small gradient in capillary pressure is imposed for a flow quantification. For unsaturated moisture storage and transport in porous building materials, Islahuddin and Janssen [5] have developed a hygric pore-scale simulator comprising the coexisting liquid and vapour phases of water, enabling numerical simulation of hygric properties.

Unfortunately, building materials often have a very wide spectrum of pore sizes, ranging from nanometers to millimeters. The presence of multiple pore scales in the studied sample can severely influence its storage and transport properties [6]. Therefore, the applied PNMs need to incorporate information over these different length scales. PNMs have the advantage that they are inherently scale-invariant, i.e. this technique can be applied to any length interval for which the pore structure has been experimentally observed and analyzed. In the past maximum balls algorithms have succeeded to cross different length scales [7,8]. In this method the largest inscribed spheres (maximal ball) that touch matrix surfaces are searched. The maximal balls fill the entire void space measuring the local apertures in pore central spaces and irregular corners. To define the topology, maximal balls are merged into clusters. Locally, the largest ball will identify the pore body, while the smallest balls, which belong to more than one family, are used to define the pore throats.

Hence, PNMs have the potential to resolve the problems related to simulate fluid flow in complex pore structures, such as building materials. This paper focusses on the generation of two datasets depicting the pore space of brick at two different spatial scales: one with micro- and one with nanometer resolutions. Further research is however necessary to arrive at a PNM incorporating information over the several length scales of the pore structure. For both scales PNM's are derived

\footnotetext{
* Corresponding author: steven.claes@kuleuven.be
} 
from a 3D image and stochastically generated based on this information. In the first part of the paper the focus is placed on the generation of these datasets. In the results and discussion section the stochastic generation algorithm is explained as well as the pitfalls related to it.

\section{Materials and imaging techniques}

Ceramic bricks used in masonry constructions are an excellent example of a building material with a multiscale pore network. In this study $4 \mathrm{~mm}$ diameter samples are drilled out of a Vandersanden Robusta brick. These bricks have a density of $1818 \mathrm{~kg} / \mathrm{m}^{3}$ and a total open porosity of $32.6 \%$ [9].

In Figure $1 \mathrm{~A}$ the pore size distribution obtained using mercury intrusion is depicted. Pore sizes range from $\mu \mathrm{m}$ to $\mathrm{nm}$ scale. The histogram shows a continuous distribution of pore sizes, which makes it difficult to use different techniques independently. Currently only indirect imaging techniques such as MIP or NMR can cover such a broad range of pore sizes. It is however not straightforward to define a PNM for this material based on the data provided by these techniques. In order to obtain 3D images of the entire spatial range two imaging techniques are used in this study: micro-CT for the micrometer scale and FIB-SEM for the nanometer scale. Based on this curve an open micrometer scale porosity of around $15 \%$ and open nanometer porosity of $17 \%$ is assumed. The micrometer scale porosity contains pores with a diameter above $3 \mu \mathrm{m}$, while for the nanometer scale porosity the smallest visible pores have a diameter of $30 \mathrm{~nm}$.

To capture the larger pores, micro-CT has proven to be an excellent tool. It allows to visualize the internal structure of objects in a non-destructive way. Hence, the heterogeneity of sample components as well as the porosity network may be characterized in a qualitative way down to a resolution of $1 \mu \mathrm{m}$ [10]. It is important to notice that although the spatial resolution is $1 \mu \mathrm{m}$, pore characteristics such as volume, radius and shape can only be accurately described if the pore radius is bigger then 5 times the spatial resolution. Hence the smallest pores captured in this dataset have a radius of $5 \mu \mathrm{m}$.

The images used in this study are taken by a GE nanotom scanner with the following parameters: $80 \mathrm{kV}$ and $160 \mu \mathrm{A}$ and a spatial resolution of $1.2 \mu \mathrm{m}$ (Figure 1B). The larger pores have a heterogenous spatial distribution in the sample and are connected by smaller pores. However, although these smaller pores are clearly visible in the slice in Figure $1 \mathrm{~B}$ the resolution is not sufficient enough to accurately describe them. The pore space in these slices is segmented using a dualthreslholding creating a binary image dataset [10]. Using an image labeling operation, the visible open porosity can be selected.

To image the lower end of the pore size range, scanning electron microscopy (SEM) has proven to be a useful and established technique for extracting 2D images of the microstructure of building materials such as concrete and stone. However it does not provide information about the third spatial component.
The combination of Focused Ion Beam (FIB) and SEM resulting in FIB-SEM enables this technique to give 3D information. It is important to note that, contrary to CT based imaging methods FIB-SEM is destructive [11]. The images are taken on a Helios Nanolab 660/G system. To obtain the best quality of images the "through the lens" detector is used in backscattered-electron mode (BSE). In this mode the electrons are generated due to elastic collisions with the atoms of the sample which causes a change in their trajectory (the billiard ball model). Because building materials are non-conductive materials charging and drift pose huge threats during imaging. Although the generation region of BSE mode is larger than that of secondary electrons resulting in a poorer spatial resolution, they are less influenced by charge up and specimen irregularities due to their larger energy.

To perform the serial sectional procedure, the sample is placed at the eucentric height, so that the imaging plane (x-y-directions) can be scanned with the electron beam under an angle of 52。 without changing sample position (Figure 1C). During the acquisition of the image stack, the imaging plane is moving step by step in $\mathrm{z}$-direction due to the sequential ion-milling.

The aim of serial sectioning is to produce a regular stack of images, which can directly be transformed into a voxel-based data volume. For this purpose, the thickness of eroded layers should have similar dimensions to the pixel resolution in the imaging plane (i.e., $10 \mathrm{~nm}$ ). Therefore, the stepwise erosion should be repeated with high precision at a constant $\mathrm{z}$-step size. Because the acquisition of hundreds of images lasts for 20 hours or even longer, drift can become significant. Without correction, the drift in z-direction causes distortions in the reconstructed 3D-microstructure. In contrast, drift components in $\mathrm{x}$ - and $\mathrm{y}$-directions are automatically compensated during the off-line data processing with image alignment (Figure 1D).

Pore network models are used since the late 1950's to represent the complex pore space of the studied sample by constructing a network of pore bodies and throats with idealized geometries. The network extraction process typically comprises two parts: splitting up the pore space representation into discrete elements and subsequently measuring the geometric properties of each network element that will be used in the modeling. Usually, these include the inscribed radius, the length, the volume and some sort of shape-describing parameter for each network element.

In order to transform the image data into special and topological property lists the maximum ball method is applied [7]. 
A
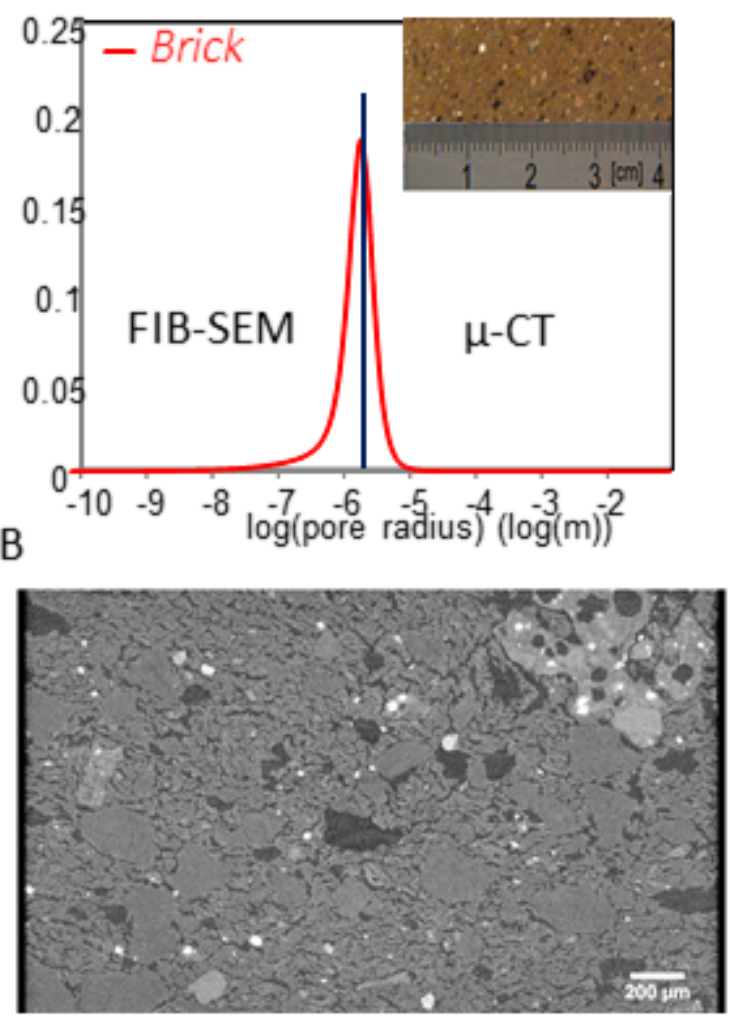

C

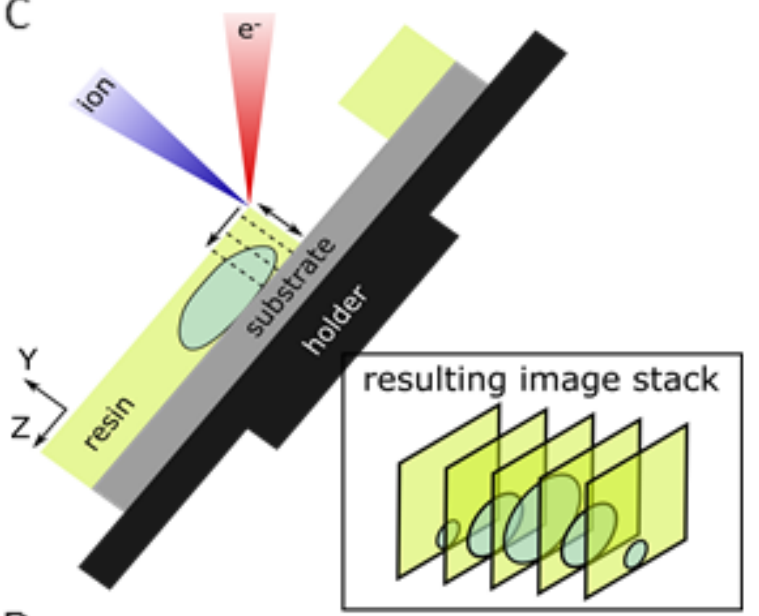

$\mathrm{D}$

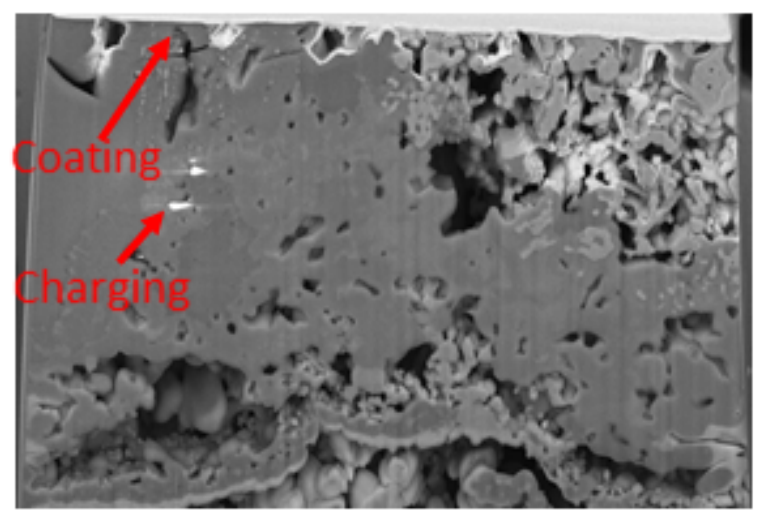

Fig. 1. A) Pore size distribution of the Vandersande brick obtained by MIP; B) Example of a micro-CT slice; C) schematic overview of the FIB-SEM setup. D) Example of a FIB-SEM slice.

\section{Stochastic network generation}

The stochastically generated networks recreate the pore space of the studied material based on input data such as distributions of pore size, throat size and connectivity. The generated networks can be arbitrarily large and are therefore not limited by the size of the original image or network. They also have the potential to be able to combine different networks of different scales.

In the past several workflows have been suggested to generate such network models. They typically progressively build on each other, as they all have the same fundamental structure but each research group tried to incorporate an additional level of complexity to improve the overall simulations. Ioannidis et al. [12] extracted pore and throat size distributions and pore connectivity information from binary images of thinsections. Starting with regular cubic lattices, they removed nodes and bonds in order to match the desired average coordination numbers corresponding to the simulated porous media. Cubic pores and rectangular throats were then distributed on the remaining nodes and bonds. Subsequently, Arns et al. [13] offered a better method of generating networks, by matching the coordination number distribution instead of just the average coordination number. Finally, Idowu et al. [14] developed tools to preserve the morphological properties of the network. Hence, they no longer require a cubic lattice, by assessing the following three observations: the minimum and maximum distances between two pores are decided by the user, each throat radius should be smaller than the pores it connects and two adjacent pores cannot overlap. They also allowed to incorporate a first degree of correlation between the building blocks of the network by letting the pore size determine the throat sizes that are connected to them.

In our approach all fundamental information about the pore network such as the inscribed radius, volume and shape factor of pore bodies and throats as well as the coordination number of the pore bodies, is gathered and a statistical distribution is fitted on each of these parameters. Copulas, functions that describe dependencies among variables, are used to this goal, as they provide a way to create distributions for correlated multivariate data. Using a copula, a multivariate distribution can be defined by specifying marginal univariate distributions and choosing a particular copula to define a correlation structure between the distributions. Bivariate distributions, as well as distributions in higher dimensions, are possible. Because of their flexibility these functions have become popular for data analysis in recent years.

The entire simulation algorithm can be described by the following steps:

1) Assign the desired dimensions to the random network. Place an equivalent number of pores as the original material within this volume. Deliberately the number of pores is used and not the porosity parameter as this allows to use the overall porosity as a quality control of the model. 
2) Generate the appropriate number of mass centers used to define the pore bodies. By using a Sobol set of coordinates a more uniform distribution in the entire simulated volume is obtained, resulting in a more accurate volume distribution fit (Figure 2A).

3) Check if the minimum and maximum distances defined by the user are respected.

4) Assign a correlated set of geometrical information and coordination number to each pore (Figure 2B). Check if any overlap of pore volumes occurs.

5) Define connections between pores by starting with the largest one and connect it with the $\mathrm{i}$ nearest pores, with $\mathrm{i}$ equal to the coordination number. For each of the connecting pores, the remaining number of connections is decreased by one.

6) To each connection a weight is assigned based on the radius of the two pore bodies it connects. The smallest throat radius along with other correlated geometrical information is assigned to the branch with the smallest weight.

7) Generate an equivalent number of inlet and outlet connections comparable to the original sample. The properties of these throats are also fitted on the original in and outlet data.

\section{Results and discussion}

The micro-CT and FIB-SEM images are segmented and the pore space is transformed into a PNM using the maximal ball approach developed by Blunt et al. [8]. As explained in section 2 due to technical aspects and resolution of both imaging techniques different volumes with different resolutions are captured. In this study 1 micrometer scale volume of $750 \times 750 \times 750$ voxels with a resolution of $1.2 \mu \mathrm{m}$ is analysed as well as three nanometer scale volumes $(500 \times 500 \times 500)$ with a resolution of $10 \mathrm{~nm}$. This approach allows to assess if substantial differences in pore topology and properties exist between the two scales. Because at the nanometer scale the selected volumes are smaller, the selection of three volumes allows to compare the obtained results and to assess the influence of the representative elementary volume. Of the three sub-volumes, volumes 1 and 2 have open porosity values close to the expected value of 17 $\%$, respectively 17.83 and $18.58 \%$. Volume 3 is situated in a more porous area and has an open porosity of $43.62 \%$. In these datasets the following independent parameters are present for pore bodies: volume, inscribed radius and shape factor as well as the number of connections with neighbouring pores (coordination number $(\mathrm{CN})$ ). For the throats, the volume, radius and shape factor are calculated as well as each throat length. The histogram of throat radii as well as their fitted distributions are shown in Figure 3A. The coordination numbers are fitted with the following discrete distributions: Binomial, Geometric and Poisson. The other parameters are fitted with continuous distributions such as: Normal, Exponential, Gamma, Logistic,
Rayleigh, Beta, Lognormal and Weibull. Additionally, the distributions are truncated between the minimum and maximum value of the original parameter. The algorithm uses a Log-likelihood criterium to determine the best fit. This makes it possible that the same parameter is fitted by different distributions at different scales. E.g. the pore body volumes of the $\mathrm{CT}$ dataset are fitted using a beta distribution while for microscopic datasets 2 and 3 a lognormal and Weibull distribution are used. Applying different distributions has indicated that the overall stochastic generation is very sensitive to the chosen distributions.

A

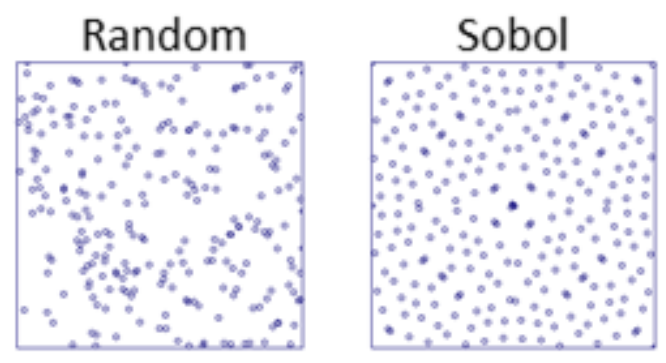

B
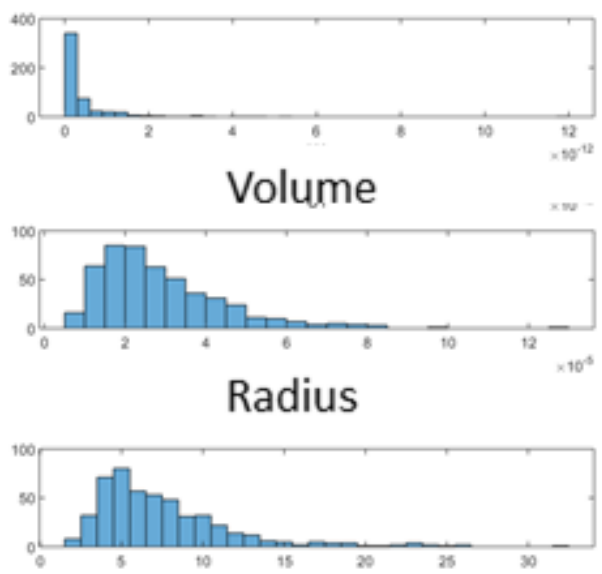

Coordination number

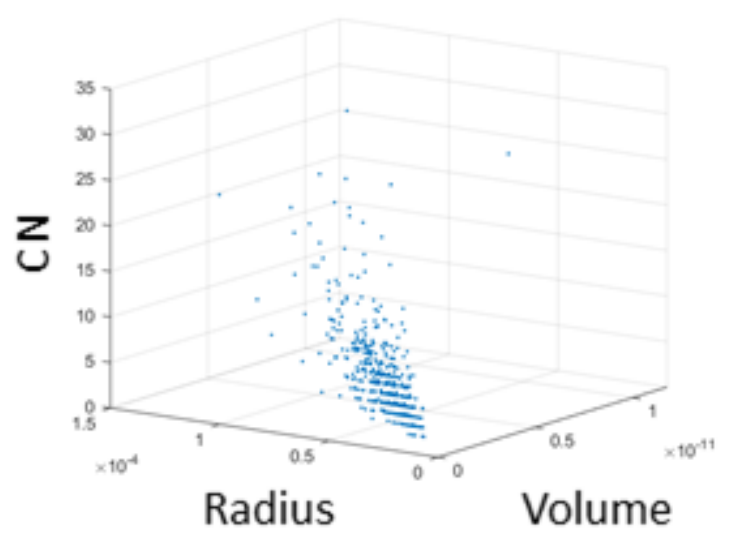

Fig. 2. A) Difference between random generated pore locations and locations generated based on a Sobol set; B) Illustration of a simulated correlated set of geometrical information of a PNM using Copulas. 
In Table 1 the correlation values for each of these parameters at the micrometer scale are gathered. As expected pore volume and radius have the highest positive correlation. In case of the throats, this correlation is significantly lower. This can be explained by the fact that the length of the throats plays a more important role in the total volume. At the nanometer scale the correlation values of the input data follow the same trends as at the micrometer scale (Table 2). However because the fitting of the data with statistical distributions is more sensitive to small changes in their respective defining parameters, the simulated correlation matrices deviate more compared to the micrometer scale.

Table 1. Correlation values at the micrometer scale level of the PNM parameters.

\begin{tabular}{|c|c|c|c|c|}
\hline Pore body & Volume & Radius & $\mathrm{CN}$ & $\begin{array}{c}\text { Shape } \\
\text { Factor }\end{array}$ \\
\hline Volume & 1 & 0.59 & 0.35 & -0.11 \\
\hline Radius & 0.59 & 1 & 0.5 & -0.34 \\
\hline $\mathrm{CN}$ & 0.35 & 0.5 & 1 & -0.45 \\
\hline Shape factor & -0.11 & -0.34 & -0.45 & 1 \\
\hline Pore throat & & & & \\
\hline Volume & 1 & 0.32 & $/$ & 0.01 \\
\hline Radius & 0.32 & 1 & $/$ & 0.003 \\
\hline Shape factor & 0.01 & 0.003 & $/$ & 1 \\
\hline
\end{tabular}

Table 2. Correlation values at the nanometer scale level of the PNM parameters of the input data and correlation values of the stochastically generated data

\begin{tabular}{|c|c|c|c|c|}
\hline Pore body & Volume & Radius & $\mathrm{CN}$ & $\begin{array}{c}\text { Shape } \\
\text { Factor }\end{array}$ \\
\hline Volume & 1 & 0.67 & 0.76 & -0.34 \\
\hline Radius & 0.67 & 1 & 0.81 & -0.58 \\
\hline $\mathrm{CN}$ & 0.76 & 0.81 & 1 & -0.56 \\
\hline Shape factor & -0.34 & -0.58 & -0.56 & 1 \\
\hline & & & & \\
\hline Volume & 1 & 0.48 & 0.56 & -0.15 \\
\hline Radius & 0.48 & 1 & 0.76 & -0.5 \\
\hline CN & 0.56 & 0.76 & 1 & -0.51 \\
\hline Shape factor & -0.15 & -0.5 & -0.51 & 1 \\
\hline
\end{tabular}

Fig. 3. A) Example of the throat radius distribution at the microscopic scale and the lognormal distribution fit; B) Histogram of the porosity values of 30 simulation of microscopic sample 3; C) Correlation between the simulated radii of pores and connecting throats at the micrometer scale; D) Histogram of the pore body volumes at the micrometer scale; E) Cumulative distribution of the pore body volumes at the microscopic scale; F) Correlation between the simulated radii of pores and connecting throats at the nanometer scale; $G$ ) Cumulative distribution of throat lengths at the nanometer scale.

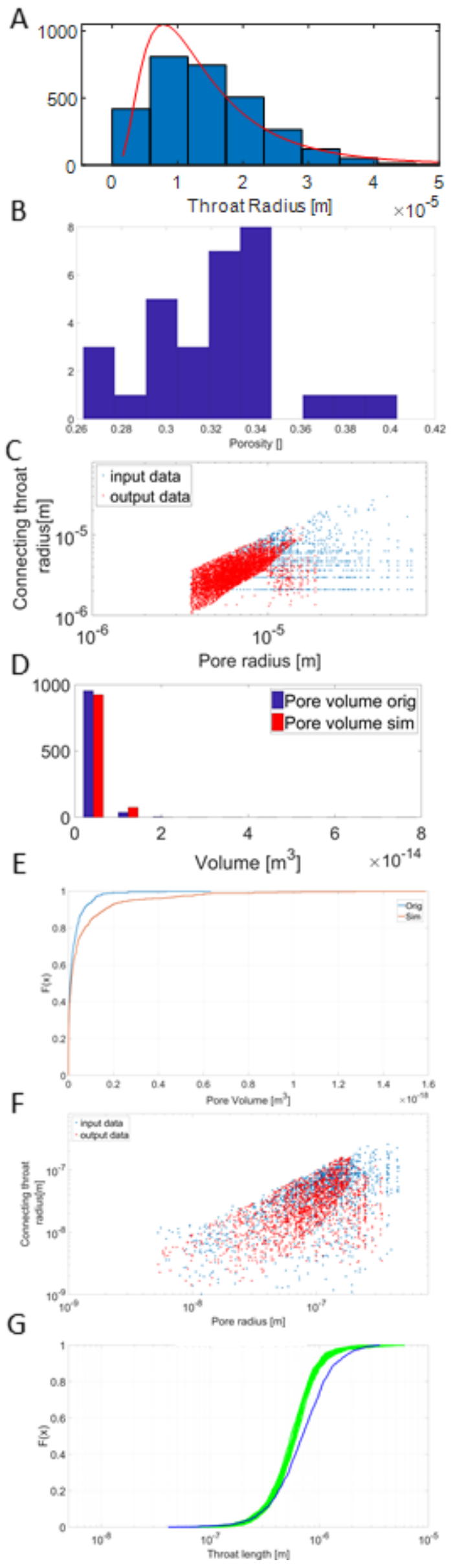


In each simulation, 30 PNMs were generated based on these input parameters. Out of this group of PNMs the simulation with a porosity value close to the average porosity value of all simulations was chosen as representative. In Figure $3 \mathrm{~B}$ the porosities of the 30 realisations of micro volume 3 are shown and a network with a porosity value of $34.3 \%$ is chosen.

In Figure 3C the connecting throat radii are plotted against the pore body radius at the micrometer scale. The precondition that the throat radius has to be smaller than the pore radius is clearly respected. The tail of the pore radii distributions is clearly different between the input and output data. However the effect on the overall hygric properties is minimal as shown by the black curves in Figure 4.

For the pore volume distributions a better fit between input and output data is obtained (Figure 3D). This is because a simulation with a similar overall open micrometer scale porosity of $15 \%$ is selected. In case of the nanometer scale volumes an accurate stochastical fitting is much harder as explained above. As expected this influences the pore volume distributions. Further research is required to identify if this is caused by resolution problems or can be influenced by the original pore network extraction.

Figure 3 F\&G allow to assess the quality of the generated connections. Subfigure F indicates a good match between the pore radii and the connecting pore radii. Additionally, the distribution of the length of the throats also matches relatively well with the original input data. Moreover, in the graph all 30 distributions are plotted indicating a stable distribution of throat lengths over all simulations.

Finally, all simulated volumes with different porosity values are subjected to invasion algorithms as developed by Islahuddin and Janssen [5]. The moisture storage and transport property curves are calculated for the entire water saturation range (Figure 4). For both parameters a good match between the original and the simulated pore network is obtained at the micrometer scale. As explained above this is due to the accurate fits for each parameter of the PNM. For the nanometer scale the differences between the original input data and the simulated results are larger. In Figure 4A the difference in moisture content between the different simulated volumes is related to their respective open porosity. The curves of the simulated volumes follow the trend of the curve of the original input volume. Although for microscopic volume 3 the difference remains large between the input value and the average volume. This can be explained due to the large spread of the porosity values of the simulations as illustrated in Figure 3B. The difference between the three volumes at the nanometer scale indicates that these volumes are probably smaller than the representative elementary volume. This is especially the case for sample 3 .

Traditionally PNMs have a certain robustness towards the different parameters from which they are made out. Idowu et al. [14] indicated that multi-phase flow properties are consistent if the original topology is well represented. In the generation of PNMs several competing properties are balanced. E.g. if a flow path is divided in more pore bodies it is anticipated to have a lower permeability because there are more resistor elements. However, this effect is balanced by smaller radii and shorter throat lengths resulting in higher permeabilities. Nevertheless, the results of this study show that PNMs are rather sensitive to the volume distribution of the pore bodies.

The results of this study are a first step towards the development of a scale invariant workflow to generate PNMs stochastically. In a next step these networks need to be combined. However due to the large difference between the scale of the micro- and nanometer datasets this could not be achieved. A too large Sobel dataset of $900 \mathrm{~GB}$ needs to be generated to form the nanometer scale porenetwork. More efficient numerical methods need to be explored in the future to solve this problem. Additionally, the initial tests indicate that a clear overlap between the micro- and nanometer scale input data are required to obtain accurate results and validate the workflow. 


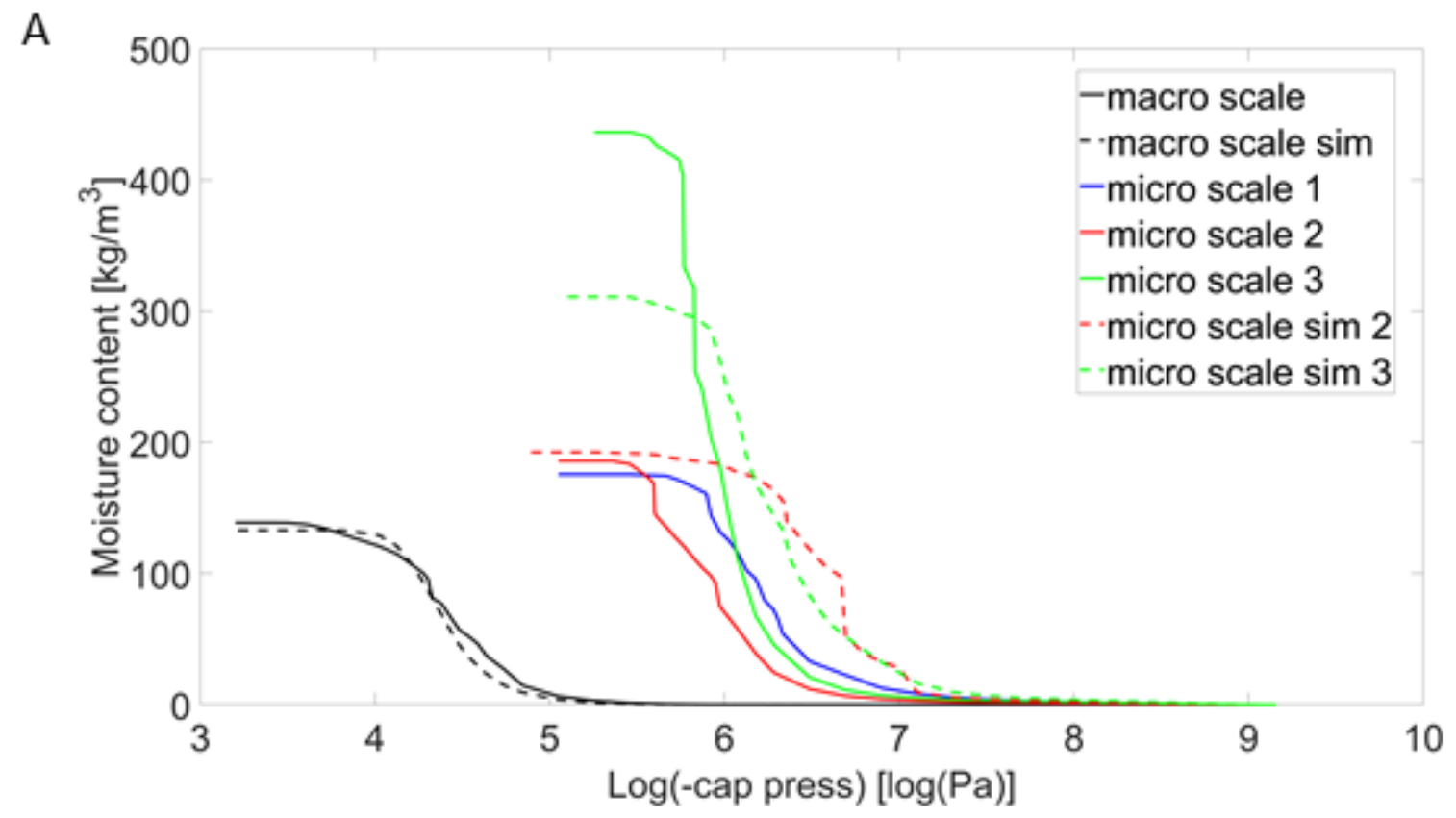

B

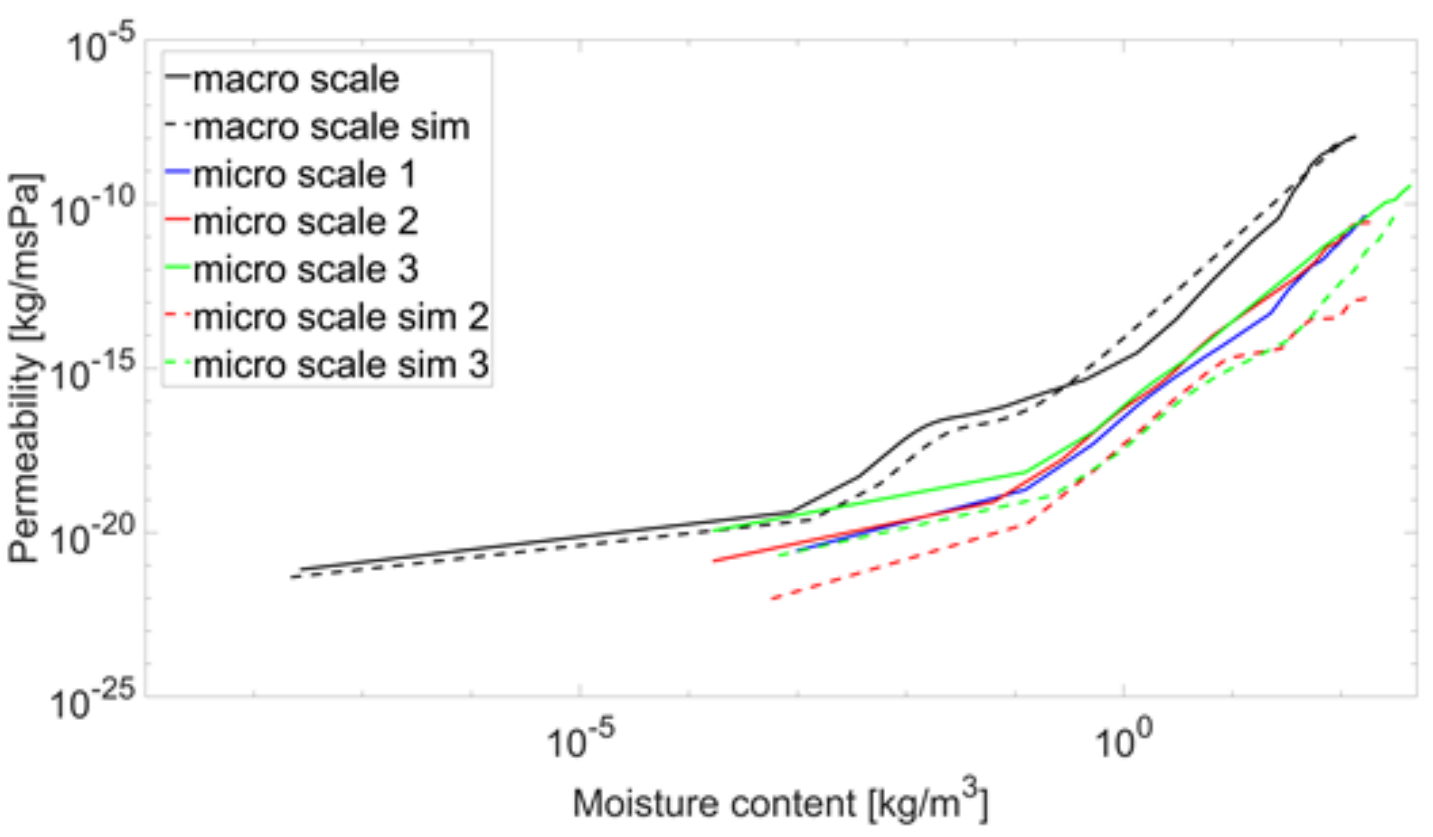

Fig. 4. Moisture retention and permeability curves for different simulation results; for the moisture permeability, the moisture content has been presented logarithmically, for the better presentation of the entire range.

\section{Conclusions}

In this paper a workflow to generate PNM at different scales based on correlated statistical distributions is presented. The results clearly indicate the scale invariance of the suggested workflow. The resulting moisture retention and transport property simulations show a good match between simulations and the original data obtained from this complicated material at the micrometer scale. For the nanometer scale further statistical and numerical methods need to be explored in the future.

Because of the broad applicability of PNMs and their versatility to incorporate different scales without exploding the calculation time required for simulating different physical properties, PNMs have the potential to resolve the problems related to simulate fluid flow in complex pore-space systems, such as building materials. In order to incorporate information of different data acquisition techniques such as direct and indirect imaging techniques, as well as the synthesis of different pore networks obtained at different spatial scales (e.g. the integration of $\mathrm{nm}, \mu \mathrm{m}$ and $\mathrm{mm}$ scale pore networks). 
This objective will form the bread and butter of our further research, as it allows to combine all available information about the pore space and come up with a full scale network which is necessary to obtain accurate simulations.

\section{References}

1. J. Carmeliet, S. Roels, J. Therm. Envelope Build. Sci.,25 (3) (2002)

2. P. H Valvatne, M., Piri, X. Lopez, M. Blunt, In Upscaling Multiphase Flow in Porous Media (p. 23-41) (2005)

3. J. T Gostick,. M. A Ioannidis,. M. W Fowler, M Pritzker J. Power Sources 173(1) (2007)

4. M. Prat, Chem. Eng. Technol. 34(7) (2011)

5. Islahuddin, M. \& Janssen, H. Transp Porous Med (2019) 130: 675

6. M. Islahuddin, H. Janssen, Energy Procedia 132 (2017)

7. D. Silin, \& T. Patzek, T. Physica A: Statistical mechanics and its applications, 371(2) (2006)

8. M. Blunt, B. Bijeljic, H. Dong, O. Gharbi, S. Iglauer, P. Mostaghimi, A. Paluszny, C. Pentland, C Advances in Water Resources, 51, pp.197-216. (2013)

9. C. Feng, \& H. Janssen, Building and Environment, 152, pp.39-49 (2019)

10. S. Claes, W. Van De Walle, M. Islahuddin, H Janssen. Journal of Building Physics 43(4) (2019)

11. L. Keller, L. Holzer, R. Wepf, P. Gasser, B. Münch, P. Marschall, P. Physics and Chemistry of the Earth, Parts A/B/C, 36(17-18) (2011)

12. M. A Ioannidis,., M. J Kwiecien,., I Chatzis,., I. F MacDonald,., F Dullien, in Paper SPE 38713, Proceedings of the SPE Annual Technical Conference and Exhibition (1997)

13. J.Y. Arns,., V. Robins, A.P. Sheppard, R.M. Sok, W.V. Pinczewski, M.A.Knackstedt Transp. Porous Media 55: p. 21-46 (2004)

14. N. A. Idowu, M Blunt Transp. Porous Media 83 (1) (2010). 